\title{
COVID-19 Associated with Severe Intracranial Hemorrhage in Previously Healthy Patients
}

\section{loan Alexandru FLORIAN ${ }^{1,2^{*}}$, Magdalena BALACI ${ }^{1}$, Teodora Larisa TIMIȘ ${ }^{3}$, Cristina Caterina ALDEA ${ }^{1}$, Laura MURESAN ${ }^{1}$, Oana Maria RADU ${ }^{1}$ and loan Stefan FLORIAN ${ }^{1,2}$}

${ }^{1}$ Clinic of Neurosurgery, Cluj County Emergency Clinical Hospital, Cluj-Napoca, Romania

${ }^{2}$ Department of Neurosurgery, Iuliu Hatieganu University of Medicine and Pharmacy, Cluj-Napoca, Romania

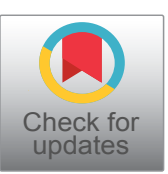

${ }^{3}$ Department of Physiology, Iuliu Hatieganu University of Medicine and Pharmacy, Cluj-Napoca, Romania

*Corresponding author: Ioan Alexandru Florian, Clinic of Neurosurgery, Cluj County Emergency Clinical Hospital, Cluj-Napoca, Romania; Department of Neurosurgery, Iuliu Hatieganu University of Medicine and Pharmacy, Cluj-Napoca, Romania

\begin{abstract}
Background: The neurological complications of COVID-19 are still under scrutiny, stroke and intracranial hemorrhages being the most commonly described events. Hemorrhagic stroke in previously healthy patients and with a normal coagulative status has only rarely been described.

Cases: We present three males aged 30,57 and 38 respectively, who suffered from intracranial hemorrhages, having been diagnosed with COVID-19, with no medical history and under no previous medication. They were all operated via external ventricular drainage under COVID-19 regulations and protection. The first two patients had died, while the third remains alive in the ICU.

Conclusion: COVID-19 may be associated with potentially fatal intracranial hemorrhage even in individuals with seemingly no risk factors.
\end{abstract}

\section{Keywords}

COVID-19, Intracranial hemorrhage, Ventricular extension, External ventricular drainage, Cerebrospinal fluid

\section{Introduction}

Ever since the emergence of the new severe acute respiratory syndrome coronavirus 2 (SARS-CoV-2) and its resulting intricate pathological picture known worldwide as COVID-19 (COronaVIrus Disease - 2019) in December 2019 in the Hubei Province of China, its repercussions on the central nervous system have been widely studied and debated [1-3]. COVID-19 was declared a pandemic by the WHO on 11 March 2020, affecting more than 83.5 million people around the world and claiming at least 1.82 million lives by the end of the year [4]. Aside from severe respiratory failure, it has been also observed that this disease has been associated with an increased incidence of encephalopathy and encephalomyelitis, ischemic and hemorrhagic stroke, as well as neuromuscular diseases $[2,3]$. Whether the SARS-CoV-2 infection triggers these manifestations directly through an unclear mechanism, or indirectly via the redistribution of healthcare sources towards the treatment and prevention of the pandemic remains to be elucidated.

The pathological pathway of the SARS-CoV-2 infection is mediated via the angiotensin-converting enzyme 2 (ACE2). This enzyme, which is found in lung alveolar cells, endothelial cells, and vascular smooth muscle cells in the human brain, had been observed to act as receptor for viral infection for other coronaviruses $[5,6]$. Patients with multiple comorbidities are at a higher risk of developing a more severe and deadly disease, however young, healthy, and active individuals are by no means safe from harm. We present the cases of three previously healthy males who contracted the SARS-Cov2 virus and presented with acute cerebral hemorrhage without an underlying cerebrovascular anomaly.

\section{Case 1}

A 30-year-old male with no relevant medical history was brought to the neurosurgical department of our

Citation: FLORIAN IA, BALACI M, TIMIȘ TL, ALDEA CC, MUREȘAN L, et al. (2021) COVID-19 Associated with Severe Intracranial Hemorrhage in Previously Healthy Patients. Neurosurg Cases Rev 4:061. doi. org/10.23937/2643-4474/1710061

Accepted: February 25, 2021; Published: February 27, 2021

Copyright: (C) 2021 FLORIAN IA, et al. This is an open-access article distributed under the terms of the Creative Commons Attribution License, which permits unrestricted use, distribution, and reproduction in any medium, provided the original author and source are credited. 


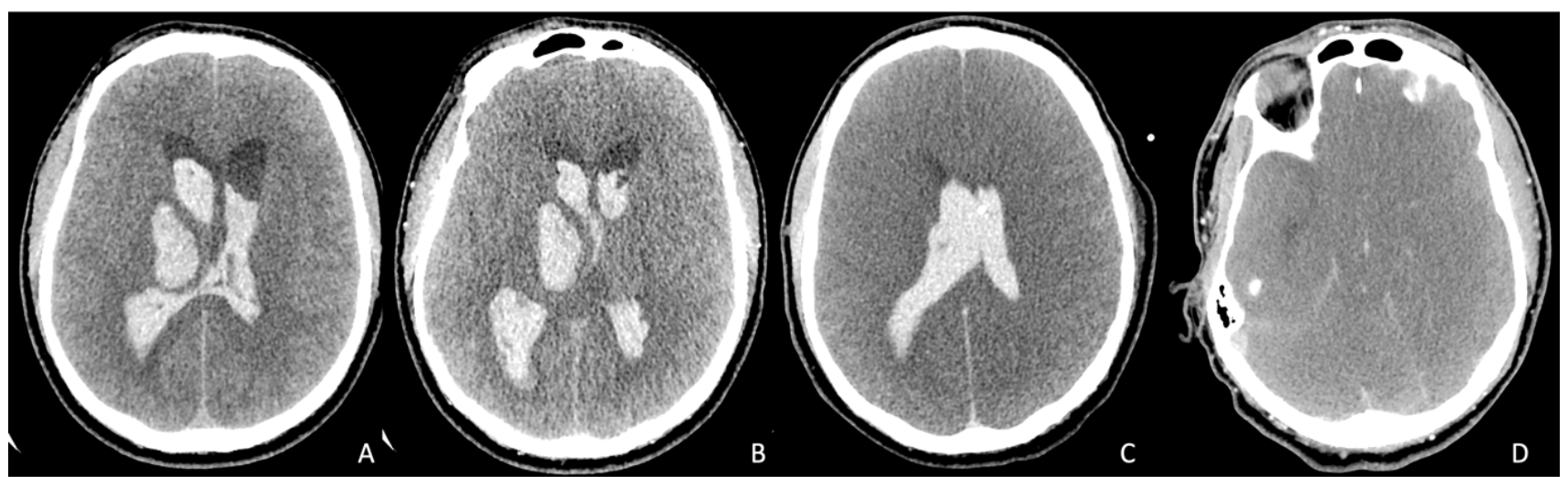

Figure 1: Head Computed tomography (CT) scans of case 1. A) Initial non-contrasted CT scan showing an acute intraparenchymal hemorrhage in the right thalamus that has extended into the ventricles; B) CT angiography (CTA) demonstrating no vascular anomalies at the level of the hematoma; C) Postoperative CT scan showing the placement of the external ventricular drainage catheter inside the lateral ventricles, a slight decrease in ventricular volume, and marked diffuse cerebral edema; D) CTA of the Circle of Willis showing no cerebral perfusion, characteristic of brain death.

hospital in an emergency setting due to sudden loss of consciousness. The patient's family disclosed that he had been complaining of severe headaches in the past three days, which did not respond to analgesics. The neurological exam at admission revealed a comatose patient with a GCS of $4(\mathrm{M} 2, \mathrm{~V} 1,01)$, fixed dilated pupils, being intubated and mechanically ventilated. Initial CT scan revealed a right thalamo-mesencephalic intraparenchymal hematoma, significant tetraventricular inundation and obstructive hydrocephalus, as well as a moderate amount of subarachnoid hemorrhage in the right Sylvian Fissure, in the Frontal lobe, and in the posterior fossa. CTA (Figure 1) did not highlight any vascular anomalies such as an arteriovenous malformation (AVM) or an intracranial aneurysm (IA). As per our protocol, we took the patient to the OR via the isolated COVID-19 circuit and performed an emergency EVD on the left side and the insertion of a right jugular central venous catheter under rigorous protection from infection with the Coronavirus. CSF was hypertensive and mixed with fresh blood.

He tested positive for SARS-CoV-2 infection using a nasopharyngeal swab and was transferred to our COVID-19 ICU compartment. He was initially stable, with a tendency for bradycardia that responded to Atropine. His neurological status remained critical, and the control CTA performed on the first postoperative day showed the reduction of ventricular volume, the absence of flow in the internal carotid arteries, as well as marked diffuse cerebral edema. CT scans of the lungs showed ground glass opacities in the inferior right lobe, consistent with theCOVID-19 aspect, as well as thin pleural collections located at the base of the lungs bilaterally. The EVD drained an average of $100 \mathrm{~mL}$ of CSF per day. He became hemodynamically unstable on the third postoperative day, requiring vasoactive support with Norepinephrine to maintain a MBP > $65 \mathrm{mmHg}$. On the sixth day after surgery, he suffered from a cardiac arrest that did not respond to CPR (according to the ERC
2015 protocol) and was pronounced dead by an anesthesiologist.

\section{Case 2}

This 57-year-old male was transferred to our department from another county for acute motor deficit on the right side, expressive aphasia, and agitation that culminated with the loss of consciousness. Apart from a sudden and unprecedented bout of hypertension leading to this incident, this patient had no relevant medical history. Upon arrival, he was sedated under Fentanyl ( -5 on the Richmond Agitation-Sedation Scale), intubated and mechanically ventilated, mildly hypothermic (35.8 ${ }^{\circ} \mathrm{C}$ ), and his pupils were myotic due to sedation. CT scan showed a left thalamic hematoma with tetraventricular inundation and dilation, whereas CTA (Figure 2) did not uncover an underlying cerebrovascular anomaly. Respecting our clinical protocol, the COVID-19 surgical circuit was initiated, a right EVD being inserted, releasing blood laden CSF under increased pressure.

He tested positive for COVID-19 by PCR from nasopharyngeal swab and was placed in our dedicated ICU compartment. After EVD insertion, his sedation was interrupted to allow extubation, with little success. He presented spontaneous eye movement but did not respond to commands and developed bouts of hypertension. Concurrently, a triple association therapy of ACE inhibitors, Urapidil and Nicardipine was initiated, lasting for six days. Afterwards, blood pressure correction was no longer required. Control CT scan on the first postoperative day showed the proper placement of the EVD, as well as a decrease in ventricular volume.

His clinical and neurological status remained unchanged during the first eight days after intervention. Approximately $200 \mathrm{~mL}$ of CSF were drained on daily average. Control head CT scan at 1 week after surgery no longer showed signs of intraventricular hemorrhage, and the hematoma had almost fully resorbed. On day 9 he developed a fever, and the EVD was removed due to 


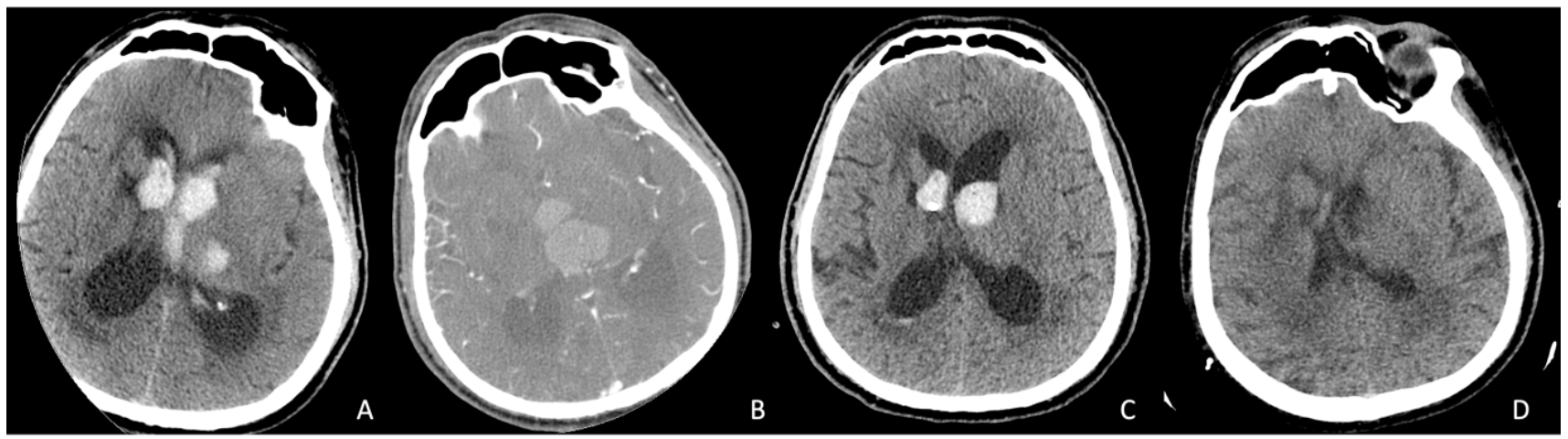

Figure 2: Head Computed tomography (CT) scans of case 2. A) Initial non-contrasted brain CT revealing the acute intraparenchymal hematoma in the left thalamus that has extended into the ventricles; B) Preoperative CT angiography (CTA) demonstrating a small deep vessel inside of the hematoma, with no traces of a malformation or aneurysm; C) Postoperative CT scan revealing the shrinkage of the intraventricular hemorrhagic extension and the placement of the external ventricular catheter inside the right lateral ventricle; D) Control non-contrasted CT scan at 1 week showing the absence of blood in the ventricles.

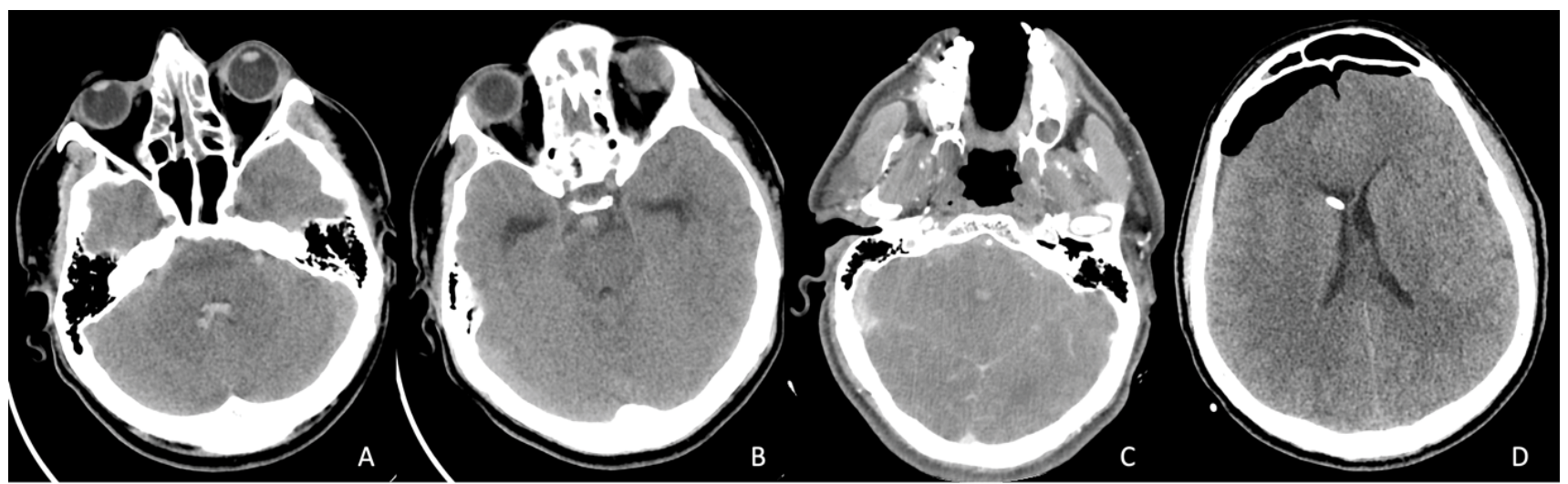

Figure 3: Head Computed tomography (CT) scans of case 3. (A,B) Initial Non-contasted CT scan demonstrating the acute subarachnoid hemorrhage anterior to the brainstem and inside the $4^{\text {th }}$ ventricle; (C) CT angiography (CTA) that did not show any aneurysms or vascular malformations of the basilar artery and its branches; (D) postoperative CT scan showing the placement of the external ventricular catheter inside the right lateral ventricle, as well as a moderate amount of pneumocephalus after trephination.

blockage. On day 13 he began to show signs of severe respiratory failure, associated with hemodynamic instability needing norepinephrine correction. Pulmonary $\mathrm{CT}$ scans at this stage revealed significant condensations spread diffusely in both lungs, as well as a thick bilateral pleural collection. He suffered from severe multiple organ failure on day 20, culminating with cardiac arrest and asystole that did not respond to CPR. He was pronounced dead by the on-call anesthesiologist.

\section{Case 3}

This 38-year-old male diagnosed with COVID-19 three weeks prior to presentation had no previous medical history. Upon admission in another center, he complained of headache, nausea and vomiting, followed by a rapid decline in consciousness and increased agitation, then culminated by generalized seizures. He was transferred to our department after the initial CTA (Figure 3) showed a pretruncal nonaneurysmal subarachnoid hemorrhage that spread into the fourth ventricle and occipital horns of the lateral ventricles, causing obstructive hydrocephalus. No intracranial vascular le- sions could be identified. Neurological examination revealed a GCS of 13 (M6, V4, O3), no discernible motor deficit, no lesions of the cranial nerves, the presence of increased intracranial pressure syndrome, and reactive pupils. Rapid COVID-19 antigen test performed just before transfer was positive. Prothrombin time and INR (international normalized ratio) were slightly increased, although not as much as to preclude surgery. Therefore, we initiated the surgical COVID-19 circuit and performed the insertion of an EVD on the right side. CSF was tinted red by fresh blood and under significant pressure.

Postoperatively, his evolution was mildly favorable, with the amelioration of the neurological status (GCS of 15 on postoperative day 2) and headache. Control CT scan disclosed the correct placement of the EVD, shrinkage of the ventricles, mild diffuse cerebral edema and no signs of an additional hemorrhage. He presented an episode of marked difficulty while breathing on postoperative day 3 , for which the anesthesiology team decided to intubate. The pulmonary CT scan was evocative of COVID-19 (ground-glass opacities in both lungs), howev- 
Table 1: Summary of patient characteristics.

\begin{tabular}{|c|c|c|c|}
\hline & Patient 1 & Patient 2 & Patient 3 \\
\hline Age $(y)$ & 30 & 57 & 38 \\
\hline Gender & Male & Male & Male \\
\hline Presenting symptoms & $\begin{array}{l}\text { Severe headache for } \\
3 \text { days, LOC }\end{array}$ & $\begin{array}{l}\text { Hypertensive bout, Acute } \\
\text { right-side motor deficit, Broca's } \\
\text { aphasia, Agitation, LOC }\end{array}$ & $\begin{array}{l}\text { Headache, Nausea, Vomiting, } \\
\text { Altered consciousness, } \\
\text { Agitation, seizures }\end{array}$ \\
\hline Fever on admission & No & No (hypothermia) & No \\
\hline Presence of seizures & No & No & Yes, Generalized \\
\hline Initial GCS & 4 (M2 V1 O1) & Sedated & 13 (M6 V4 O3) \\
\hline Pupils on admission & Fixed, Mydriatic & Myotic (sedation) & Reactive, Isochoric \\
\hline Ventilated on arrival & Yes & Yes & No \\
\hline Brain CT findings (admission) & $\begin{array}{l}\text { Intraparenchymal } \\
\text { hematoma, No } \\
\text { vascular anomaly, } \\
\text { Involvement of all } \\
\text { ventricles, Secondary } \\
\text { SAH }\end{array}$ & $\begin{array}{l}\text { Intraparenchymal hematoma, } \\
\text { No vascular anomaly, } \\
\text { Involvement of all ventricles }\end{array}$ & $\begin{array}{l}\text { Pretruncal nonaneurysmal } \\
\text { SAH, Involvement of } 4^{\text {th }} \\
\text { ventricle }\end{array}$ \\
\hline Type and Location of ICH & $\begin{array}{l}\text { Right thalamic, } \\
\text { Panventricular } \\
\text { extension, } \\
\text { Right sylvian SAH }\end{array}$ & $\begin{array}{l}\text { Left thalamic, Panventricular } \\
\text { extension }\end{array}$ & $\begin{array}{l}\text { Pretruncal } \mathrm{SAH}, 4^{\text {th }} \text { ventricle } \\
\text { extension }\end{array}$ \\
\hline Initial lung CT findings & $\begin{array}{l}\text { Ground glass } \\
\text { opacities in the } \\
\text { inferior right lobe, } \\
\text { Thin pleural fluid } \\
\text { collection bilaterally }\end{array}$ & $\begin{array}{l}\text { Ground glass opacities } \\
\text { affecting both lungs, Bilateral } \\
\text { fluid collection approx. } 1 \mathrm{~cm} \\
\text { thick }\end{array}$ & $\begin{array}{l}\text { Ground glass opacities in the } \\
\text { lower lobes }\end{array}$ \\
\hline COVID-19 PCR positivity date & 24.11.2020 & 09.11 .2020 & 30.11 .2020 \\
\hline Days intubated & 6 & 20 & 7 (interrupted) \\
\hline $\begin{array}{l}\text { Anticoagulant/antiplatelet (after } \\
\text { admission) }\end{array}$ & No & Fraxiparine & $\begin{array}{l}\text { Fraxiparine } \\
\text { clexane }\end{array}$ \\
\hline Intervention for $\mathrm{ICH}$ & Right EVD & Right EVD & Right EVD \\
\hline Concomitant infections & None detected & None detected & Pulmonary \\
\hline CSF pressure and Aspect (surgery) & $\begin{array}{l}\text { Increased pressure, } \\
\text { Blood red }\end{array}$ & Increased pressure, Blood red & $\begin{array}{l}\text { Increased pressure, Slightly } \\
\text { tinted red }\end{array}$ \\
\hline \multicolumn{4}{|c|}{ Lab Findings on Admission (measure units) } \\
\hline WBC $\left(10^{9} / L\right)$ & $19.52(4-10)$ & $13.54(4-10)$ & $16.68(4-10)$ \\
\hline $\mathrm{RBC}\left(10^{12} / \mathrm{L}\right)$ & $5.78(4.5-5.5)$ & 5.31 & 4.82 \\
\hline HGB (g/dL) & 16.5 & 15.7 & 14.3 \\
\hline HCT (\%) & 49.9 & 48.8 & 42.6 \\
\hline $\operatorname{MCV}(f L)$ & 86.3 & 92.0 & 88.4 \\
\hline $\mathrm{MCH}(\mathrm{pg})$ & 28.5 & 29.7 & 29.6 \\
\hline $\mathrm{MCHC}(\mathrm{g} / \mathrm{dL})$ & 33.0 & 32.2 & 33.4 \\
\hline RDW-SD (fL) & 40.9 & 41.5 & 41.0 \\
\hline RDW-CV (\%) & 13.7 & 13.0 & 13.1 \\
\hline PLT (10\%/L) & 375 & 175 & 253 \\
\hline PDW (fL) & 15.9 & $16.3(10.1-16.1)$ & 15.7 \\
\hline MPV (fL) & 9.3 & 11.7 & 9.3 \\
\hline PCT (\%) & $0.351 \%(0.17-0.32 \%)$ & $0.204 \%$ & $0.237 \%$ \\
\hline P-LCR (\%) & $21.0 \%$ & $36.6 \%$ & $20.2 \%$ \\
\hline NEUT\% & $83.8 \%(30-75 \%)$ & $81.5 \%(30-75 \%)$ & $85.9 \%(30-75 \%)$ \\
\hline NEUT\# $\left(10^{9} / \mathrm{L}\right)$ & $16.35(1.5-6.6)$ & $11.03(1.5-6.6)$ & $16.04(1.5-6.6)$ \\
\hline LYMPH\% & $8.6 \%(20-45 \%)$ & $11.0 \%(20-45 \%)$ & $5.9 \%(10-45 \%)$ \\
\hline LYMPH\# & 1.68 & 1.49 & 1.10 \\
\hline
\end{tabular}




\begin{tabular}{|c|c|c|c|}
\hline MONO\% & $7.6 \%$ & $7.4 \%$ & $8.0 \%$ \\
\hline MONO\# & $1.48(0.21-0.92)$ & $1.01(0.21-0.92)$ & $1.50(0.21-0.92)$ \\
\hline $\mathrm{EO} \%$ & $0.0 \%(1-5 \%)$ & $0.0 \%(1-5 \%)$ & $0.1(1-5 \%)$ \\
\hline EO\# & $0.00(0.02-0.67)$ & $0.00(0.02-0.67)$ & 0.02 \\
\hline BASO $\%$ & 0.0 & $0.1 \%$ & $0.1 \%$ \\
\hline BASO\# & 0.01 & 0.01 & 0.02 \\
\hline ALAT & 45 & 24 & 26 \\
\hline ASAT & 29 & 25 & 19 \\
\hline Direct bilirubin & 0.06 & 0.15 & 0.20 \\
\hline Total bilirubin & 0.32 & 0.79 & 0.89 \\
\hline lonized calcium (mg/dL) & $4.38(4.4-5.4)$ & 4.62 & 4.47 \\
\hline Total calcium (mg/dL) & $8.77(8.8-10.6)$ & 9.02 & $8.52(8.8-10.6)$ \\
\hline Creatin kinase (U/L) & $226(<171)$ & 165 & $350(<171)$ \\
\hline CK-MB (U/L) & $29(<24)$ & 13 & 9 \\
\hline Creatinine (mg/dL) & 0.93 & $2.24(0.67-1.17)$ & $0.61(0.67-1.17)$ \\
\hline Alkaline phosphatase & 76 & 62 & 71 \\
\hline GGT (U/L) & 28 & 38 & 22 \\
\hline Glycemia (mg/dL) & $174(74-106)$ & $122(74-106)$ & 96 \\
\hline Sodium [Na] (mmol/L) & 138 & 141 & 142 \\
\hline Potassium [K] (mmol/L) & 4.47 & 4.39 & $3.47(3.5-5.1)$ \\
\hline Chloride [Cl] (mmol/L) & 105 & 105 & 106 \\
\hline LDH (U/L) & 231 & 214 & $\mathrm{~N} / \mathrm{A}$ \\
\hline C-reactive Protein (mg/dL) & 0.34 & $1.17(<0.5)$ & $10.77(<0.5)$ \\
\hline Urea (mg/dL) & 30 & $64(17-43)$ & 24 \\
\hline APTT (sec.) & 21.3 & 21.7 & 20.8 \\
\hline PT (sec.) & 12.8 & 12.8 & $16.2(10.5-15)$ \\
\hline Prothrombin index (\%) & 89.1 & 89.1 & $70.6(80-120)$ \\
\hline INR & 1.12 & 1.12 & $1.34(0.8-1.22)$ \\
\hline Outcome & Deceased & Deceased & Alive (in ICU) \\
\hline
\end{tabular}

Concerning lab test results, they are in the normal range of their parameters according to our laboratory, except where specified (bolded and normal range in parentheses). ALAT: Alanine aminotransferase; APTT: Activated partial thromboplastin time; ASAT: Aspartate transaminase; BASO\#: Basophil count; BASO\%: Basophil percentage; CK-MB: Myocadiac creatin kinase; COVID-19: Coronavirus disease 2019; CSF: Cerebrospinal fluid; CT: Computer tomography; EO\#: Eosinophil count; EO\%: Eosinophil percentage; EVD: External ventricular drainage; Fl: Femtoliters; GCS: Glasgow coma score; GGT: Gamma-glutamyl transferase; HCT: Hematocrit; HGB: Hemoglobin; ICH: Intracranial hemorrhage; INR: International normalized ratio; LDH: Lactate dehydrogenase; LOC: Loss of consciousness; LYMPH\#: Lymphocyte count; LYMPH\%: Lymphocyte percentage; MCH: Mean corpuscular hemoglobin; MCHC: Mean corpuscular hemoglobin concentration; MCV: Mean corpuscular volume; MONO\#: Monocyte count; MONO\%: Monocyte percentage; MPV: Mean platelet volume; N/A: Not applicable; NEUT\#: Neutrophil count; NEUT\%: Neutrophil percentage; P-LCR: Platelet large cell ratio; PCR: Polymerase chain reaction; PCT: Procalcitonin; PDW: Platelet distribution width; pg: Picograms; PLT: Platelet count; PT: Prothrombin time; RBC: Red blood cell count; RDW-SD: Red blood cell distribution width standard deviation; RDW-CV: Red blood cell distribution width coefficient of variation; SAH: Subarachnoid hemorrhage; U/L: Units per liter of blood; WBC: White blood cell count; y: Years.

er PCR test returned negative. Furosemide, dexamethasone and mannitol therapy was initiated. Extubation occurred on the following day, the patient being aware, cooperative, and stable. However, after 5 days, the EVD began to show signs of blockage, the patient becoming somnolent. Control CT scan confirmed our suspicion by showing enlarged ventricles. As such, the EVD was replaced on day 6 . Afterward, the patient regained awareness. Subsequent COVID-19 PCR tests all had negative results, the patient being transferred to our regular ICU compartment. CSF tests revealed an infection with Klebsiella specie, for which antibiotic lavage via the EVD was started. Tracheobronchial suction culture was positive for Acinetobacter baumannii. He currently remains in the ICU, with a decreased sense of awareness.

Table 1 presents a summary of patient characteristics, as well as the lab test results at admission.

\section{Discussion}

Although catastrophic brain hemorrhages have been described in older or critically ill patients with COVID-19 [7-13], those given prophylactic anticoagulation for COVID-19 [14-17], or cases under ECMO (extracorporeal membrane oxygenation) for the respiratory distress- 
caused by this disease [16,18-21], such events in young otherwise healthy individuals with no preexisting cerebrovascular lesions or not receiving the aforementioned therapies remain rare. Patients on ECMO have formerly demonstrated high rates of intracranial hemorrhage due to the complex adjustment of anticoagulation needed to maintain appropriate oxygenation, even in the presence of other viral respiratory diseases [22-24]. According to the study by Melmed, et al., anticoagulation was strongly correlated with intracranial hemorrhage and mortality from such an event [25]. Cerebral venous sinus thrombosis has also been described as a cause for hemorrhagic stroke [26-30], however, as shown on CTA, this was not the case in any of our patients.

Microbleeds in critically individuals who are intubated and ventilatedhave been described in patients infected with SARS-CoV-2, and it can occur in a typical locations [31-34]. One of the possible explanations was hypoxemia, as respiratory failure was more pronounced and lengthier in these patients [34]. Another possible reason was microangiopathy, as suggested bythe greater rise in D-dimers and tendency toward thrombotic events. A third feasible justification was the presence of kidney failure.Still, all of the cases described with microhemorrhages presented neurological symptoms after hospitalization and mechanical ventilation for a longer period of time and were all under prophylactic or therapeutic anticoagulation.

Cerebral microangiopathy has been proposed as a possible outcome of COVID-19 involving the brain, possibly because of the development of antiphospholipid antibodies $[35,36]$. There is a possibility that at least one of the patients in our report could be explained by such a complication. Nevertheless, such tests could not be performed at our institution.

In their review, Cheruiyot and associates observed that most of the patients suffering from ICH in COVID-19 were male and elderly, harboring comorbidities the most common of which was systemic hypertension, and half of the patients were under anticoagulation [37]. They also noted that while intraparenchymal hemorrhages were the most frequent variety, intraventricular hemorrhage was the rarest. They also calculated an overall mortality of $48.6 \%$ in these patients. Similarly, in a more a recent review of the literature, Freiman, et al. gathered a total of 35 patients with COVID-19 suffering from intracranial hemorrhage, out of which 5 had no known pre-existing risk factors for hemorrhagic stroke [6]. However, as the authors inferred, there were as of yet too few cases to provide adequate data for an etiopathogenetic hypotheses. Additionally, because of the need of anticoagulant therapy when treating these patients for COVID-19, it is difficult to estimate the involvement of the viral infection in itself toward the predisposition of intracranial hemorrhage.

Bao, et al. reported a previously healthy 38-year-old male with COVID-19 who suffered a sudden loss of consciousness brought on by a large left frontal hematoma [38]. Neither did hehave risk factors for such an event, and nor were there any vascular lesions discovered during surgery. Al-olama, et al. described the case of a 36-yearold male patient with SARS-CoV-2 meningoencephalitis that was complicated with a right frontal intraparenchymal hematoma associated with $\mathrm{SAH}$ in the sylvian fissure on the same side and a thin acute subdural hematoma affecting the same regions [39]. No underlying diseases or risk factors were described, and nor were there any vascular lesions found on CTA. After evacuating the subdural hematoma, PCR test of the collected fluid was positive for the coronavirus. The neurotrophic properties of the virus associated with its ACE2 binding capability may explain the direct structural damage of the blood-brain barrier (BBB), as well as the injury to the smaller vessels via systemic hypertension $[40,41]$. It has also been observed that SARS-CoV-2 infection induces coagulopathy, triggers a hypercoagulative state, and disrupts endothelial function as substantiated by heightened fibrinogen, D-dimer, and factors VIII and von Willebrand, with only a mild declinein platelet count [42-45]. The pattern observed is dissimilar to that of disseminated intravascular coagulopathy (DIC) met in sepsis. This would explain the increased incidence ischemic stroke events in COVID-19 patients; however, it does not elucidate how other individuals suffer from hemorrhages under a normal coagulative state. Additionally, despite the fact that COVID-19 can lead to a secondary immune thrombocytopenia [45], this was not present in our patients, who all had normal platelet counts.

None of the patients in our series received anticoagulation prior to the onset of $\mathrm{ICH}$, and neither were they on any chronic medication interfering with this process. For this reason, our cases present an interesting and possibly new insight into the neurological implications of COVID-19. Furthermore, to the best of our knowledge, we reported the youngest patient to have died of a cerebral hemorrhage associated with COVID-19 and without any prior medical history (Case 1).

\section{Limitations}

Limitations include the small size of the series, the fact that D-dimers were not evaluated, and that MRI scans could not be performed to better visualize the presumed cerebral angiopathy. A detailed autopsy would have definitely been useful to understand the microscopic cerebrovascular disturbances that ensued in these patients. Regrettably, due to the COVID-19 status and the lack of proper equipment (as recommended by the $C D C$ ) to perform autopsies on these patients, such a procedure was not feasible at the time. Despite the fact that the exact mechanism of this dreaded complication remains uncertain, we are optimistic that the widespread immunization against this dreaded virus may avertmany moreinstances such as these from occurring. 


\section{Conclusions}

These three cases show that COVID-19 can be associated with fatal intracranial hemorrhage in previously healthy individuals with no identifiable cerebrovascular pathology, although the exact mechanism of this manifestation remains to be clarified. Symptoms non-specific to COVID-19 such as severe and unremitting headache, sudden neurological deficit or epilepsy, and alterations in the state of consciousness should be a warning sign for a possible cerebral involvement. Early intervention and evacuation of the ensuing intraventricular hemorrhage via an EVD did nothing to improve the clinical progression in two of these patients, as the damage to the deep and eloquent structures of the brain was already severe. As treatment after the fact remains challenging and with no guarantee of success, widespread vaccination against this dreaded virus may prevent further such cases from occurring.

\section{Patient Consent}

The families of the patients presented in this report have given their written permission to the publication of this manuscript under the rights of patient confidentiality.

\section{Ethical Approval}

The ethical review board of the treating hospital gave their consent to the publication of these case reports.

\section{Acknowledgements}

The authors would like to express their gratitude toward the entire personnel of the hospital wherein this work was conducted, for their tireless work in combating this devastating pandemic. We would like to pass on good wishes to the survivors of COVID-19, who believed in us and never gaveup hope. Last, but not least, our prayers go out to the grieving families of the patients we were unfortunately unable to save.

\section{Conflict of Interest}

The authors declare that there are no conflicts of interest regarding the writing or publishing of this manuscript.

\section{Author Contributions}

All authors contributed equally to the writing and editing of this manuscript.

\section{Funding}

No funding was received.

\section{References}

1. Guan WJ, Ni ZY, Hu Y, Liang WH, Ou CQ, et al. (2020) Clinical characteristics of coronavirus disease 2019 in China. N Engl J Med 382: 1708-1720.

2. Berlit P, Bosel J, Gahn G, Isenmann S, Meuth SG, et al. (2020) Neurological manifestations of COVID-19" - guideline of the German society of neurology. Neurol Res Pract.
3. Mao L, Jin H, Wang M, Hu Y, Chen S, et al. (2020) Neurologic manifestations of hospitalized patients with coronavirus disease 2019 in Wuhan, China. JAMA Neurol 77: 683-690.

4. Roser M, Ritchie H, Ortiz-Ospina E, Hasell J (2020) Coronavirus pandemic (COVID-19).

5. Li W, Moore MJ, Vasilieva N, Sui J, Wong SK, et al. (2003) Angiotensin- converting enzyme 2 is a functional receptor for the SARS coronavirus. Nature 426: 450-454.

6. Fraiman P, Godeiro C Jr, Moro E, Cavallieri F, Zedde M (2020) COVID-19 and Cerebrovascular diseases: A systematic review and perspectives for stroke management. Front Neurol.

7. Carroll E, Lewis A (2020) Catastrophic intracranial hemorrhage in two critically III patients with COVID-19. Neurocrit Care 26: 1-5.

8. Li J, Long X, Zhu C, Hu S, Lin Z, et al. (2020) A case of COVID-19 pneumonia with cerebral hemorrhage. Thromb Res 193: 22-24.

9. Krzystanek E, Gawryluk J, Arkuszewski M (2020) Fatal intracranial haemorrhage in a hypertensive patient with atrial fibrillation and coronavirus disease 2019. Neuroradiol J.

10. Benger M, Williams O, Siddiqui J, Sztriha L (2020) Intracerebral haemorrhage and COVID-19: Clinical characteristics from a case series. Brain Behav Immun 88: 940-944.

11. Gogia B, Fang X, Rai P (2020) Intracranial hemorrhage in a patient with COVID-19: Possible explanations and considerations. Cureus 12: e10159.

12. Fayed I, Pivazyan G, Conte AG, Chang J, Mai JC (2020) Intracranial hemorrhage in critically ill patients hospitalized for COVID-19. J Clin Neurosci 81: 192-195.

13. Mousa-Ibrahim F, Berg S, Od TPDetola O, Teitcher M, Ruland S (2021) Intracranial hemorrhage in hospitalized SARS-CoV-2 patients: A case series. J Stroke Cerebrovasc Dis 30: 105428.

14. Ghani MU, Kumar M, Ghani U, Sonia F, Abbas SA (2020) Intracranial hemorrhage complicating anticoagulant prophylactic therapy in three hospitalized COVID-19 patients. J Neurovirol 26: 602-604.

15. Dogra S, Jain R, Cao M, Bilaloglu S, Zagzag D, et al. (2020) Hemorrhagic stroke and anticoagulation in COVID-19. J Stroke Cerebrovasc Dis 29: 104984.

16. Rothstein A, Oldridge O, Schwennesen H, Do D, Cucchiara BL (2020) Acute cerebrovascular events in hospitalized COVID-19 patients. Stroke 51: e219-e222.

17. Khattar NK, Sharma M, McCallum AP, Oxford BG, Zeb H, et al. (2020) Intracranial hemorrhage in a young COVID-19 patient. Interdiscip Neurosurg 22: 100878.

18. Zahid MJ, Baig A, Galvez-Jimenez N, Martinez N (2020) Hemorrhagic stroke in setting of severe COVID-19 infection requiring extracorporeal membrane oxygenation (ECMO). J Stroke Cerebrovasc Dis 29: 105016.

19. Heman-Ackah SM, Su YS, Spadola M, Petrov D, Chen $\mathrm{HI}$, et al. (2020) Neurologically devastating intraparenchymal hemorrhage in COVID-19 patients on extracorporeal membrane oxygenation: A case series. Neurosurgery 87: E147-E151.

20. Usman AA, Han J, Acker A, Olia SE, Bermudez C, et al. (2020) A case series of devastating intracranial hemorrhage during venovenous extracorporeal membrane oxygenation for COVID-19. J Cardiothorac Vasc Anesth 34: 3006-3012. 
21. Motoie $\mathrm{R}$, Akai M, Kitahara $\mathrm{T}$, Imamura $\mathrm{H}$, Tanabe $\mathrm{T}$, et al. (2020) Coronavirus disease 2019 complicated by multiple simultaneous intracerebral hemorrhages. Intern Med 59: 2597-2600.

22. Davies A, Jones D, Bailey M, Beca J, Bellomo R, et al (2009) Extracorporeal membrane oxygenation for 2009 Influenza $\mathrm{A}(\mathrm{H} 1 \mathrm{~N} 1)$ acute respiratory distress syndrome. JAMA 302: 1888-1895.

23. Chaves RCF, Rabello Filho R, Timenetsky KT, Moreira FT, Vilanova LCS, et al. (2019) Extracorporeal membrane oxygenation: A literature review. Oxigenacao por membranaextracorporea: Revisao da literatura. Rev Bras Ter Intensiva 31: 410-424.

24. Chow FC, Edlow BL, Frosch MP, Copen WA, Greer DM (2011) Outcome in patients with H1N1 influenza and cerebrovascular injury treated with extracorporeal membrane oxygenation. Neurocrit Care 15: 156-160.

25. Melmed KR, Cao M, Dogra S, Zhang R, Yaghi S, et al. (2020) Risk factors for intracerebral hemorrhage in patients with COVID-19. J Thromb Thrombolysis 24: 1-8.

26. Dakay K, Cooper J, Bloomfield J, Overby P, Mayer SA, et al. (2020) Cerebral venous sinus thrombosis in COVID-19 infection: A case series and review of the literature. J Stroke Cerebrovasc Dis 30: 105434.

27. Tu TM, Goh C, Tan YK, Leow AS, Pang YZ, et al. (2020) Cerebral venous thrombosis in patients with COVID-19 infection: A case series and systematic review. J Stroke Cerebrovasc Dis 29: 105379.

28. Mowla A, Shakibajahromi B, Shahjouei S, Borhani-Haghighi A, Rahimian N, et al. (2020) Cerebral venous sinus thrombosis associated with SARS-CoV-2; A multinational case series. J Neurol Sci 419: 117183.

29. Argiro R, Cirelli C, Sgreccia A, Del Sette B, Da Ros V (2020) Cerebral hemorrhage related to vein thrombosis in Covid-19 patients in different Italian hospitals: View point for clinical and imaging implications. J Neurol Sci 416: 117023

30. Hemasian H, Ansari B (2020) First case of Covid-19 presented with cerebral venous thrombosis: A rare and dreaded case. Rev Neurol (Paris) 176: 521-523.

31. Vattoth S, Abdelhady M, Alsoub H, Own A, Elsotouhy A (2020) Critical illness-associated cerebral microbleeds in COVID-19. Neuroradiol J 33: 374-376.

32. Gupta NA, Lien C, Iv M (20200 Critical illness-associated cerebral microbleeds in severe COVID-19 infection. Clin Imaging 68: 239-241.
33. Fitsiori A, Pugin D, Thieffry C, Lalive P, Vargas MI (2020) COVID-19 is Associated with an unusual pattern of brain microbleeds in critically III patients. J Neuroimaging 30: 593-597.

34. Lersy F, Willaume T, Brisset JC, Collange O, Helms J, et al. (2020) Critical illness-associated cerebral microbleeds for patients with severe COVID-19: Etiologic hypotheses. J Neurol 21: 1-9.

35. Shoskes A, Migdady I, Fernandez A, Ruggieri P, Rae-Grant A (2020) Cerebral microhemorrhage and purpuric rash in COVID-19: The case for a secondary microangiopathy. J Stroke Cerebrovasc Dis 29: 105111.

36. Zhang Y, Xiao M, Zhang S, Xia P, Cao W, et al. (2020) Coagulopathy and antiphospholipid antibodies in patients with Covid-19. N Engl J Med 382: e38.

37. Cheruiyot I, Sehmi P, Ominde B, Bundi P, Mislani M, et al. (2020) Intracranial hemorrhage in coronavirus disease 2019 (COVID-19) patients. Neurol Sci 3: 1-9.

38. Bao Y, Lin SY, Cheng ZH, Xia J, Sun YP, et al. (2020) Clinical features of COVID-19 in a young man with massive cerebral hemorrhage-case report. SN Compr Clin Med 23: 1-7.

39. Al-Olama M, Rashid A, Garozzo D (2020) COVID-19-associated meningoencephalitis complicated with intracranial hemorrhage: A case report. Acta Neurochir (Wien) 162: 1495-1499.

40. Baig AM, Khaleeq A, Ali U, Syeda H (2020) Evidence of the COVID-19 Virus targeting the cns: Tissue distribution, host-virus interaction, and proposed neurotropic mechanisms. ACS Chem Neurosci 11: 995-998.

41. Moriguchi T, Harii N, Goto J, Harada D, Sugawara H, et al. (2020) A first case of meningitis/encephalitis associated with SARS-Coronavirus-2. Int $\mathrm{J}$ Infect Dis 94: 55-58.

42. Qi X, Keith KA, Huang JH (2020) COVID-19 and stroke: A review. Brain hemorrhages.

43. Levi M, Thachil J, Iba T, Levy JH (2020) Coagulation abnormalities and thrombosis in patients with COVID-19. Lancet Haematol 7: e438-e440.

44. Md Noh MSF (2020) COVID-19 and cerebral hemorrhage: Proposed mechanisms. J Neuroradiol.

45. Bhattacharjee S, Banerjee M (2020) Immune thrombocytopenia secondary to COVID-19: A systematic review. SN Compr Clin Med 19: 1-11. 